\title{
Влияние осцилляции лазерного излучения на качество поверхности изделий при прямом лазерном выращивании
}

\author{
A.M. Вильданов, wildam92@mail.ru, К. Д.Бабкин, Е. В. Земляков, М.О.Гущина \\ Санкт-Петербургский морской технический университет, Санкт-Петербург, Россия
}

\begin{abstract}
В настоящее время аддитивные технологии являются одними из самых динамично развивающихся методов получения изделий. Наибольший интерес вызывает технология высокоскоростного прямого лазерного выращивания (ВПлВ), когда в зону воздействия лазерного излучения подается газопорошковая смесь, в результате образуется ванна расплава, а после затвердевания - наплавочный валик. Так появляется возможность изготавливать трехмерные изделия со сложной геометрией с высокой производительностью. В современном авиационном двигателестроении существует множество элементов, которые предпочтительнее изготавливать технологией ВПлВ, в частности, крупногабаритные изделия с толщиной стенки $\geq 3$ мм, материал Вт6. В процессе работы было выявлено, что с Гауссовым распределением пучка в тонкостенных элементах образуются несплавления по краям. Данные дефекты являются концентраторами напряжений и значительно снижают механические свойства изделий. Основными требованиями к данным деталям являются высокие эксплуатационные свойства, а именно - минимизация пор, отсутствие трещин и несплавлений как между слоями, так и по краям стенок, низкая шероховатость поверхности, чтобы уменьшить последующую механическую обработку.
\end{abstract}

\section{Effects of Beam Oscillation on Quality of Laser Metal Deposited Parts}

Vildanov A.M., wildam92@mail.ru, Babkin K.D., Zemlyakov E.V., Gushchina M.O. Saint Petersburg State Marine Technical University, Saint Petersburg, Russia

Nowadays, additive technologies are one of the most fast developing methods of obtaining products. The greatest interest is the technology of high-speed direct laser deposition (HSDLD), when a gas powder stream is fed into the laser radiation zone, as a result, a melt pool is formed, and then a layer - after hardening. Thus, it becomes possible to produce three-dimensional products with complex geometry with high productivity.

The modern aircraft engine industry has lots of elements that are more preferable to be manufactured by the HSDLD technology: large-sized products with wall thickness $\geq 3 \mathrm{~mm}$, material Ti-6Al-4V, in particular. In this work, it was found that with Gaussian beam distribution in thin element non-fusion on the edges is formed. These defects are stress concentrators and significantly reduce the mechanical properties of details. The main requirements for these parts are high performance properties, namely pore minimization, 
absence of cracks and fusions both between the layers and at the edges of the walls, low surface roughness to reduce subsequent machining.

Analyzing the possible solutions helped to find out the necessity of using a wobbler or beam shaper to achieve the required properties. As a result, wobbler was used for the research, because unlike the beam shaper, it has great functionality and the possibility of changing the wall thickness, by changing the scanning amplitude within the deposition process.

The research was carried out with the help of the laser metal deposition robotic complex, based on the fiber laser "LS-5". The complex also includes a six-axis robot manipulator, a two-axis positioner and a sealed chamber with a $6 \mathrm{~m}^{3}$ volume. The working tool is the laser welding head D30 Wobble Module of IPG Company with a three-jet nozzle.

As a result of this work, optimal parameters (laser power, velocity of process, spot size, scanning amplitude) were determine to produce a thin wall with a minimum amount of defects (absence of cracks, non-fusion in the center and at the edges of the walls) and a low roughness $\left(R_{\max } \approx 40 \mu, R a \approx 8 \mu\right)$.

Keywords: additive technologies, high-speed direct laser deposition, beam oscillation, wobbler, roughness

\section{1. ВВЕДЕНИЕ}

Титановые сплавы все больше используются в промышленности благодаря эффективному сочетанию свойств высокой удельной прочности и жаропрочности (до 400-600 들. Титановые сплавы обладают высокой коррозионной стойкостью в большинстве сред [1-4]. Механическая обработка титановых сплавов затруднена из-за низкой теплопроводности и прилипания материалов к инструменту [5]. Прямое лазерное выращивание является перспективным методом изготовления деталей с минимальной последующей механической обработкой [6-8].

Известно, что лазерный пучок имеет гауссово распределение, из-за чего в тонких элементах отсутствует проплавление по краям (рис. 1). Эти дефекты становятся концентраторами напряжений и значительно снижают механические свойства деталей.

Анализ возможных решений позволил выявить необходимость использования вобблера или формирователя луча (beam-shaper) для достижения требуемых свойств. В результате для исследования был выбран вобблер. В отличие от формирователя луча он обладает большими функциональными возможностями и позволяет варьировать толщину стенки за счет изменения амплитуды сканирования в процессе осаждения.

\section{2. ЭКСПЕРИМЕНТАЛЬНАЯ ЧАСТЬ}

\section{1. Технологический комплекс}

Исследование проводились на роботизированном комплексе для прямого лазерного выращивания, собранного на базе волоконного волоконного-

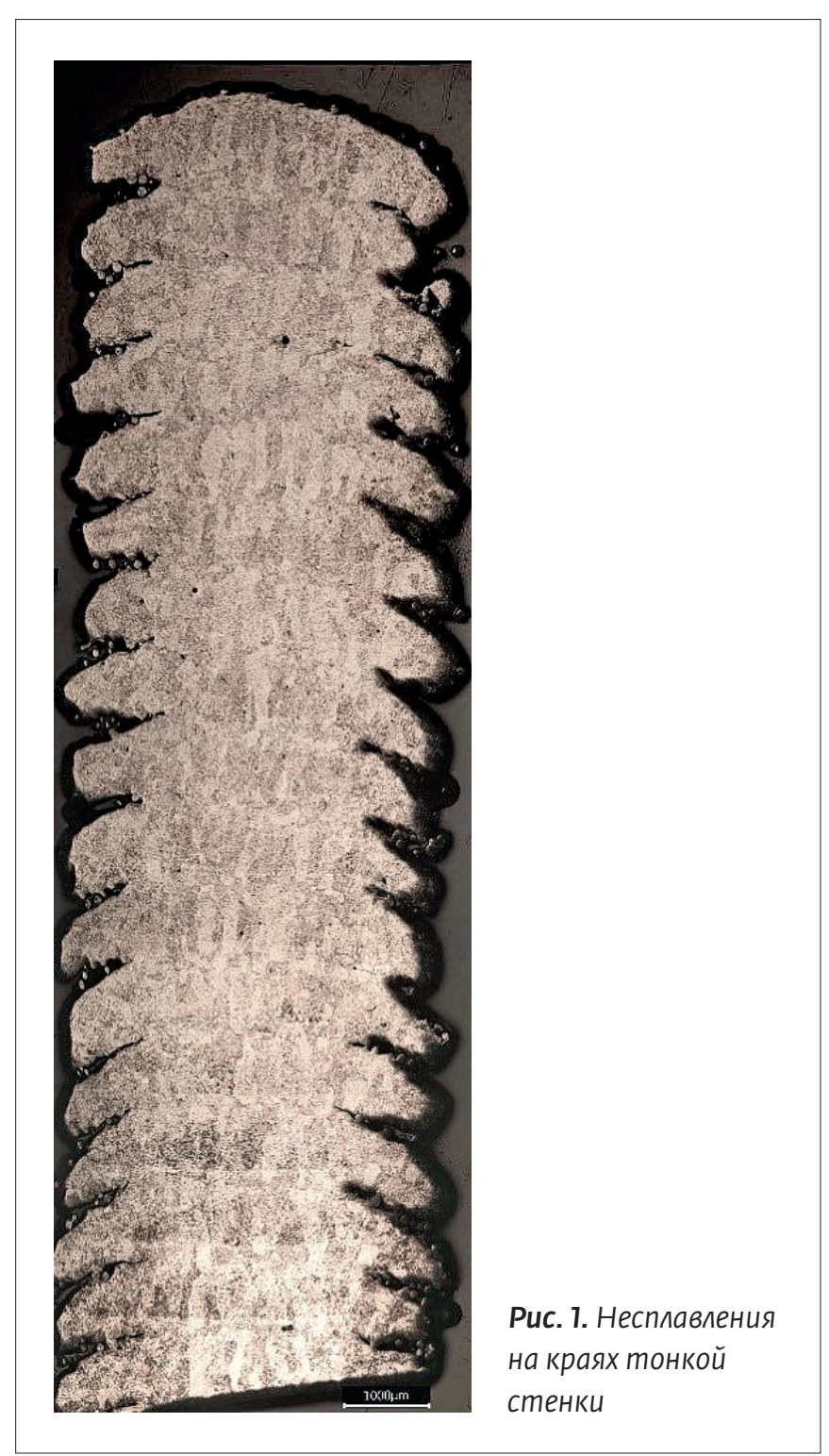




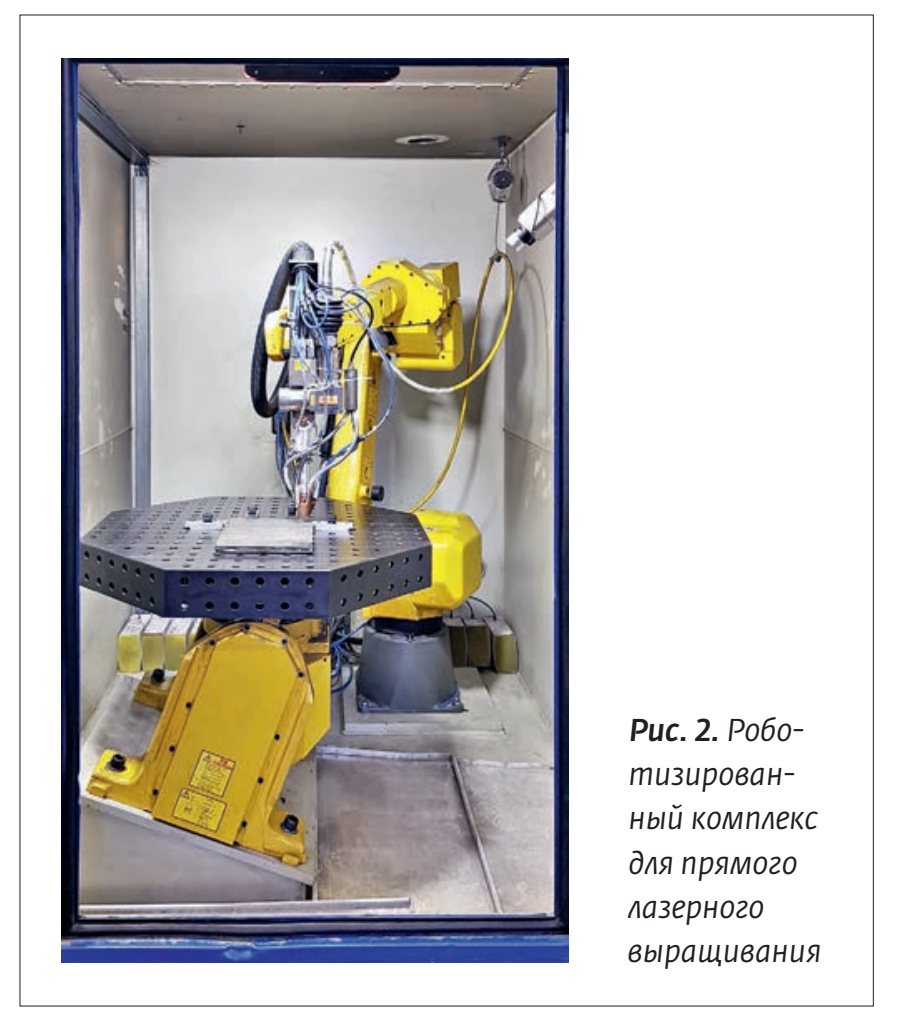

\section{2. Технологические эксперименты}

Критериями для оптимального технологического режима являются:

- толщина стенки $\geq 3,3$ мм (в авиастроении множество деталей на основе тонкой стенки $(\approx 3 \mathrm{MM}))$;

- стабильность процесса;

- минимизация дефектов (минимизация пор, несплавлений, отсутствие трещин);

- минимизация шероховатости для минимальной последующей механической обработки.

В табл. 1 представлены варьируемые технологические параметры. Габариты выращенных образцов: 40 мм длина, высота 20 мм. На рис. 4 представлена фотография процесса.

\section{3. Измерение шероховатости образцов}

После изготовления были измерены толщины образцов и их шероховатость. Для измерения шероховатости использовался контактный профилометр Hommel Tester W55 (рис. 5). Длина трассирования lt была выбрана 4,8 мм как оптимальное значение для измерения небольших образцов. иттербиевого лазера "ЛС-5". В состав установки входят: шестиосевой робот -манипулятор, двухосевой наклонноповоротный позиционер и герметичная камера объемом $6 \mathrm{~m}^{3}$. Рабочим инструментом является лазерная сварочная головка D30 Wobble Module фирмы IPG и трехструйное сопло для подачи порошка. На рис. 2 представлен роботизированный комплекс.

Сварочная головка D30 Wobble Module оснащена 2-осевым сканером (рис. 3) и позволяет осциллировать лазерный луч с максимальной амплитудой 2,5 мм и частотой 300 Гц. Оптимальной формой сканирования является линия (сканирование выполняется перпендикулярно направлению выращиваемых образцов). При линейном сканировании луч останавливается в крайних точках своей траектории [9]. Это приводит к увеличению тепловложения, что предотвращает эффект несплавления по краям.

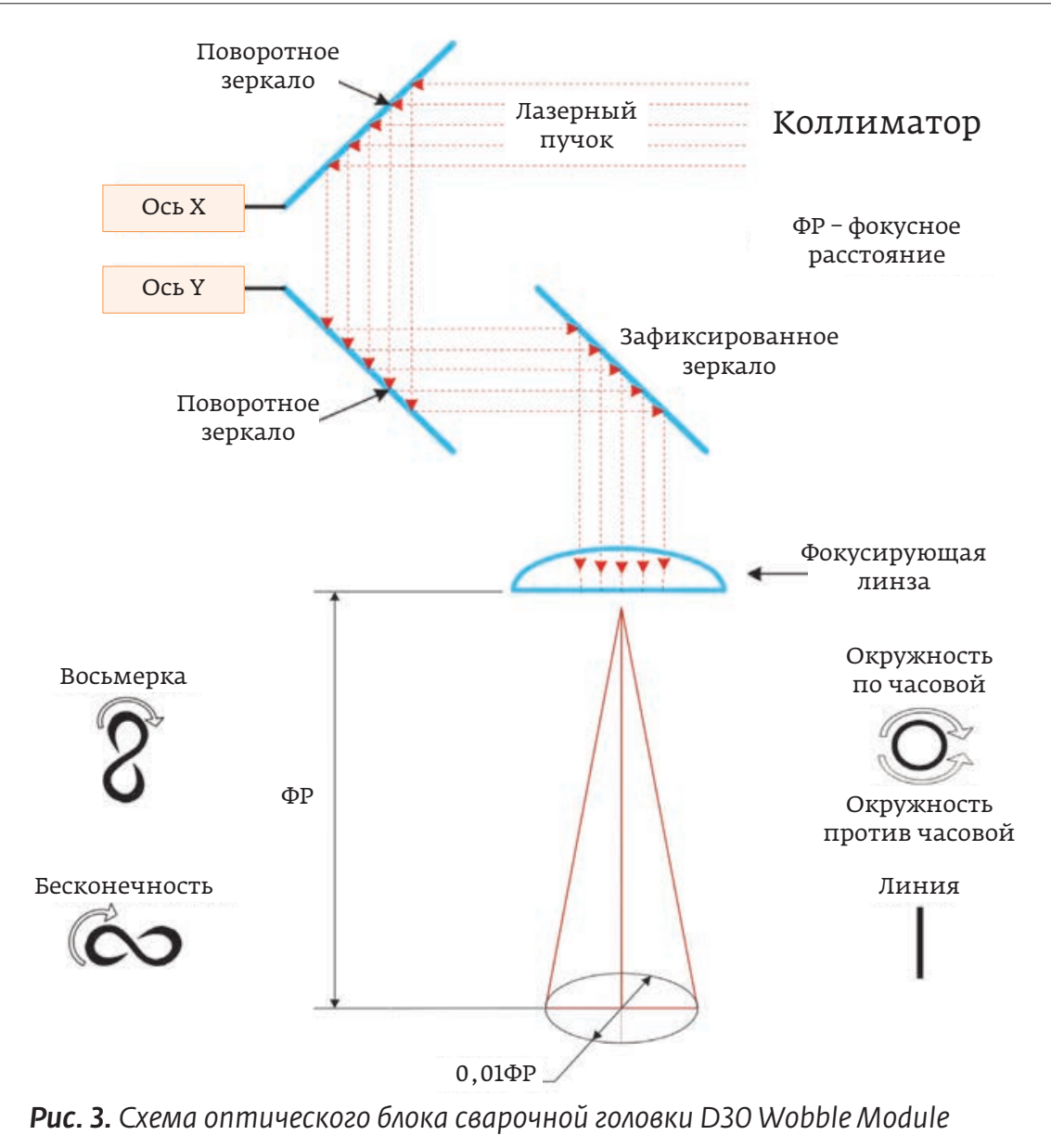

Puc. 3. Схема оптического блока сварочной головки D30 Wobble Module 


\section{ТЕХНОЛОГИИ И ТЕХНОЛОГИЧЕСКОЕ ОБОРУДОВАНИЕ}

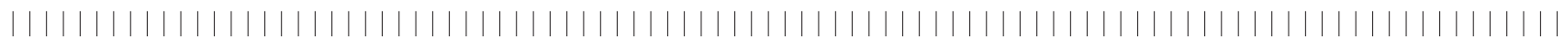

Таблица 1. Технологические параметры выращиваемых образцов

\begin{tabular}{|c|c|c|c|c|}
\hline № & $\begin{array}{c}\text { Мощность } \\
\text { лазерного } \\
\text { излучения, } \\
\text { Вт }\end{array}$ & $\begin{array}{c}\text { Скорость } \\
\text { процесса, } \\
\text { мм/с }\end{array}$ & $\begin{array}{c}\text { Диаметр } \\
\text { пучка, } \\
\text { мм }\end{array}$ & $\begin{array}{c}\text { Амплитуда } \\
\text { сканирования, } \\
\text { мм }\end{array}$ \\
\hline 1 & 1700 & 20 & 2 & 2,5 \\
\hline 2 & 1500 & 20 & 2 & 2 \\
\hline 3 & 1300 & 20 & 2 & 1,5 \\
\hline 4 & 1200 & 20 & 2 & 1 \\
\hline 5 & 2000 & 30 & 2 & 2,5 \\
\hline 6 & 1800 & 30 & 2 & 2 \\
\hline 7 & 1500 & 30 & 2 & 1,5 \\
\hline 8 & 1300 & 30 & 2 & 1 \\
\hline 9 & 1900 & 20 & 2,5 & 2,5 \\
\hline 10 & 1800 & 20 & 2,5 & 2 \\
\hline 11 & 1700 & 20 & 2,5 & 1,5 \\
\hline 12 & 1400 & 20 & 2,5 & 1 \\
\hline 13 & 2100 & 30 & 2,5 & 2,5 \\
\hline 14 & 2000 & 30 & 2,5 & 2 \\
\hline 15 & 1700 & 30 & 2,5 & 1,5 \\
\hline 16 & 1700 & 30 & 2,5 & 1 \\
\hline 17 & 2100 & 20 & 3 & 2,5 \\
\hline 18 & 2000 & 20 & 3 & 2 \\
\hline 19 & 1900 & 20 & 3 & 1,5 \\
\hline 20 & 1800 & 20 & 3 & 1 \\
\hline 21 & 2300 & 30 & 3 & 2,5 \\
\hline 22 & 2200 & 30 & 3 & 2 \\
\hline 23 & 2300 & 30 & 3 & 1,5 \\
\hline 24 & 2200 & 30 & 3 & 1 \\
\hline
\end{tabular}

Таблица 2. Результаты измерения толщины и шероховатости

\begin{tabular}{|c|c|c|}
\hline № & $\mathrm{Ra}, \mathrm{MKM}$ & Толщина стенки, мм \\
\hline 1 & 16,07 & 3,4 \\
\hline 2 & 26 & 2,9 \\
\hline 3 & 21,06 & 2,7 \\
\hline 4 & 21,71 & 2,5 \\
\hline 5 & 14,48 & 3,4 \\
\hline 6 & 23,5 & 3 \\
\hline 7 & 23,7 & 2,6 \\
\hline 8 & 25,48 & 2,4 \\
\hline 9 & 13 & 3,6 \\
\hline 10 & 11,2 & 3,4 \\
\hline 11 & 13,63 & 3 \\
\hline 12 & 23 & 2,6 \\
\hline 13 & 10,89 & 3,2 \\
\hline 14 & 12,64 & 3,1 \\
\hline 15 & 14,9 & 2,7 \\
\hline 16 & 27,48 & 2,4 \\
\hline 17 & 7,72 & 3,6 \\
\hline 18 & 16,19 & 3,4 \\
\hline 19 & 19,4 & 3 \\
\hline 20 & 23,48 & 2,7 \\
\hline 21 & 7,76 & 3,3 \\
\hline 22 & 14,48 & 3,2 \\
\hline 23 & 20,89 & 2,8 \\
\hline 24 & 24,5 & 2,5 \\
\hline
\end{tabular}

ИНФРАКРАСНЫЕ ФОТОПРИЕМНИКИ $\lambda=2-8$ мКМ

СВЕТОДИОДЫ $\lambda=3-7$ мКм

ИНФРАКРАСНЫЕ ГАЗОВЫЕ СЕНСОРЫ ПИРОМЕТРИЧЕСКИЕ ДАТЧИКИ

\section{ПРЕИМУЩЕСТВА:}

Высокая чувствительность

Низкое энергопотребление

Высокое быстродействие

Неограниченный срок службы

\section{ПРИМЕНЕНИЯ:}

Приборы газового анализа

Низкотемпературная пирометрия

Тестирование тепловизионных систем

Тепловизоры

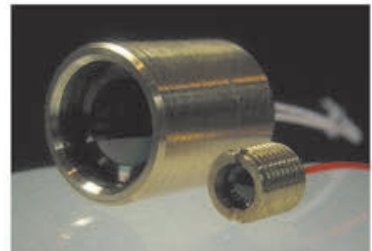

000 «ИоффеЛЕД»

www.ioffeled.com, Mremennyy@mail.ioffe.ru, +7911 2294360, Санкт-Петербург, Политехническая 26 


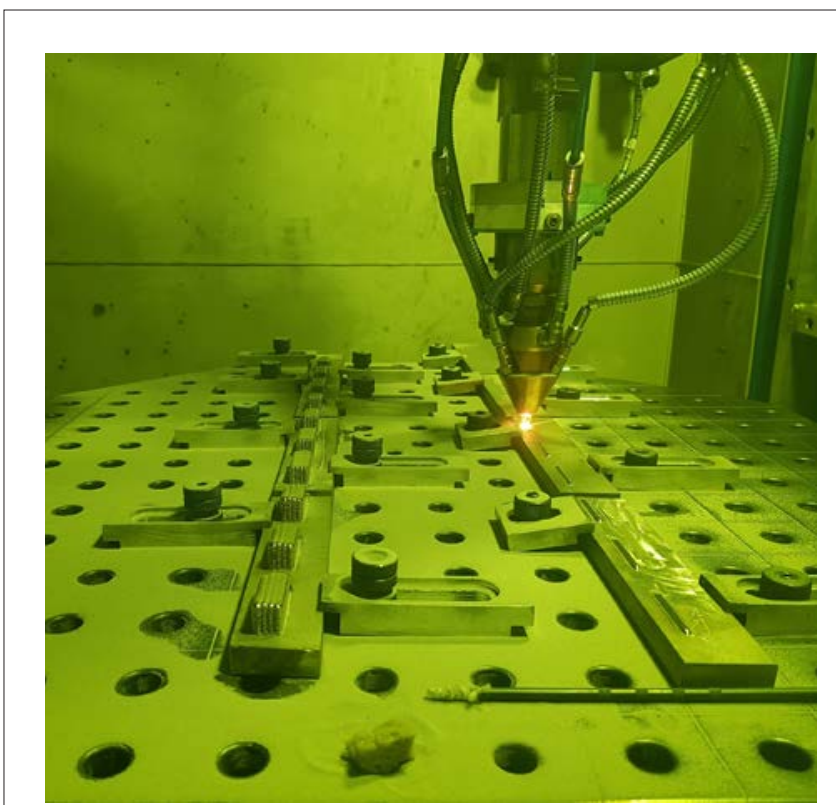

Puc. 4. Процесс прямого пазерного выращивания

Скорость перемещения щупа была установлена на 0,5 мм/с. В табл. 2 представлены результаты измерений шероховатостей и толщин изготовленных образцов. Также результаты измерений приведены в виде графиков, рис. 6.

\section{4. Анализ результатов}

Как показано на рис. 6, существует корреляция между амплитудой сканирования и шероховатостью. С увеличением амплитуды сканирования

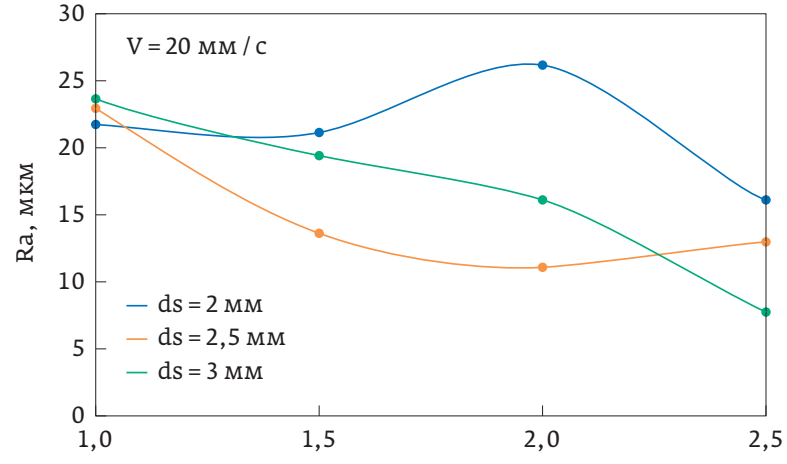

a)

Амплитуда сканирования, мм

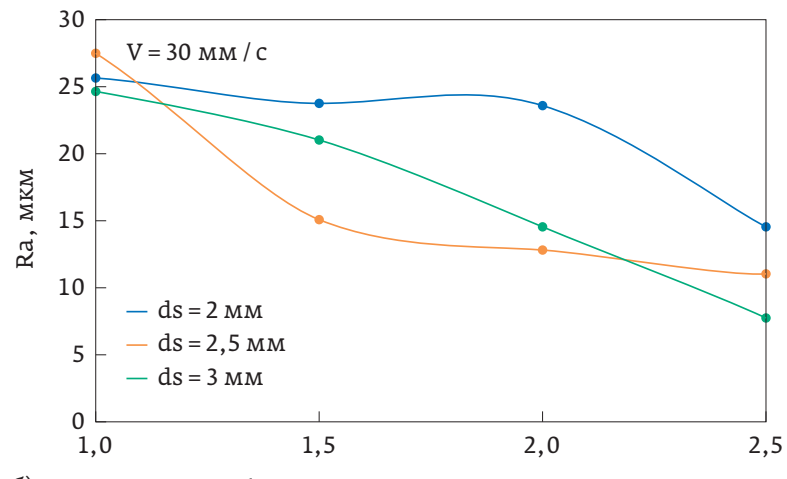

б)

Амплитуда сканирования, мм

Pис. 6. Графики зависимостей шероховатости от амплитуды сканирования (ds-диаметр пятна, мм; Ra-шероховатость поверхности, мкм; V-скорость процесса, мм/с)

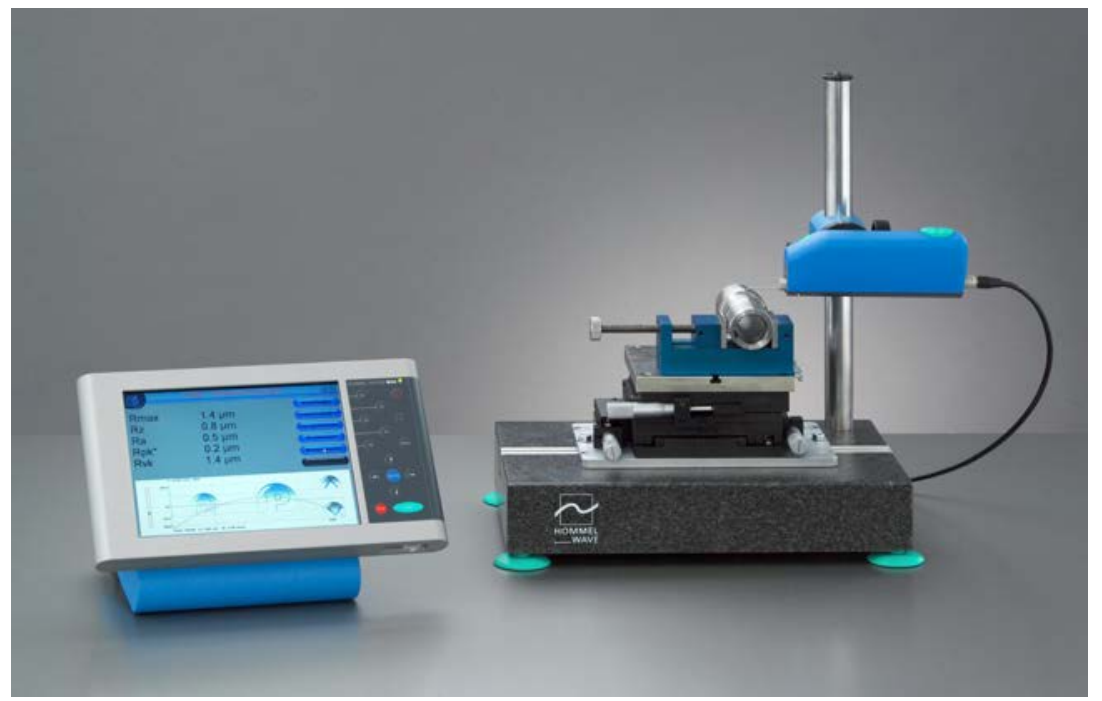

Puc. 5. Профипометр Hommel tester W55 шероховатость уменьшается. На рис. 7 показано, что крайняя точка переплавления предыдущего слоя A1 становится дальше от центра и ниже, чем точка А. Осцилляция "линией" позволяет замедлить скорость перемещения лазерного излучения в крайних точках сканирования, в результате чего происходит интенсивное тепловложение и полное переплавление подаваемого порошка с предыдущим слоем. В результате, использование сканирования приводит к отсутствию несплавлений по краям тонкой стенки, а также позволяет уменьшить шероховатость. 


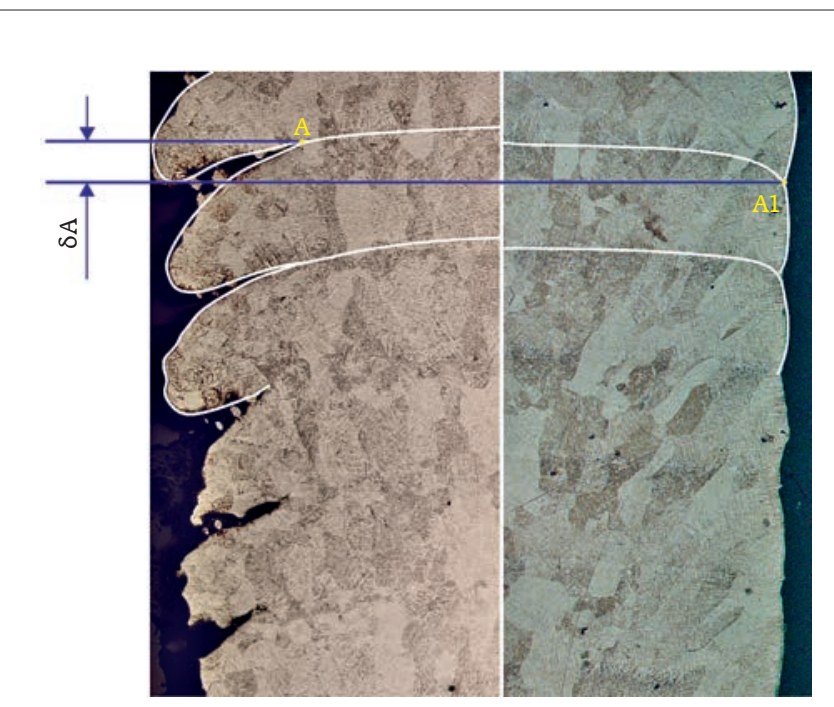

Puс. 7. Изготовленные образцы: а) без сканирования; б) со сканированием

\section{вывОДы}

В результате данной работы были проведены технологические эксперименты по изготовлению тонкой стенки с использованием вобблера. Было установлено, что дополнительное сканирование лазерного луча приводит к уменьшению шероховатости и полному переплавлению порошка на краях стенки. В результате, использование осцилляции позволяет изготавливать изделия толщиной $>3 \mathrm{MM}$ с минимальным количеством дефектов и минимальным значением шероховатости. Следующим этапом этого исследования будет изучение механических свойств изготовленных образцов.

\section{БЛАГОДАРНОСТЬ}

Работа выполнена при финансовой поддержке Министерства образования и науки Российской Федерации в рамках реализации комплексного проекта (регистрационный номер AAAA-A17-117080910109-9).

\section{ВКЛАД ЧЛЕНОВ АВТОРСКОГО КОЛЛЕКТИВА В РАБОТУ}

Идея проведения эксперимента, организация и руководство проектом: Бабкин К.Д., Земляков Е.В.; дизайн эксперимента: Бабкин К.Д., Вильданов А.М.; проведение эксперимента: Вильданов А.М.; обобщение данных и обработка результатов: Вильданов А.М., Гущина М.О. Все авторы переработали и дополнили рукопись.

\section{СПИСОК ЛИТЕРАТУРЫ}

1. Sha W, Malinov S. Titanium alloys: modelling of microstructure, properties and applications. Woodhead Publishing Limited. 2009; 598.

2. Leyens $\mathbf{C}$, Peters $\mathbf{M}$. Titanium and titanium alloys. Fundamentals and applications. Wiley-VCH Verlag $\mathrm{CmbH} \&$ C C. 2003; 514.

3. Materials properties handbook: Titanium alloys. ASM International. 2007; 788.

4. Donachie MJ. Titanium: A technical guide. Second Edition. ASM International. 2007; 216.

5. Davim JP. Machining of titanium alloys. Springer-Verlag Berlin Heidelberg. 2014; 153.

6. Gu DD, Meiners W, Wissenbach K, Poprawe R. Laser additive manufacturing of metallic components: materials, processes and mechanisms. International materials reviews. 2012;57:3:133-164.

7. G. A. Turichin, V.V. Somonov, K. D. Babkin, E. V.Zemlyakov, O. G. Klimova. High-speed direct laser deposition: technology, equipment and materials. IOP conference series: materials science and engineering. 2016; 125: 012009.

8. Klimova-Korsmik O., Turichin G., Zemlyakov E., Babkin K. Technology of high-speed direct laser deposition from Ni-based superalloys. Physics procedia. 2016;83:716-722.

9. Turichin G. A., Zemlyakov E. V., Pozdeeva E. Yu., Tuominen J., Vuoristo. Technological possibillities of laser cladding with the help of powerful fiber lasers. Metal Science and Heat Treatment, Vol. 2012; 54, Nos. 3-4, July.

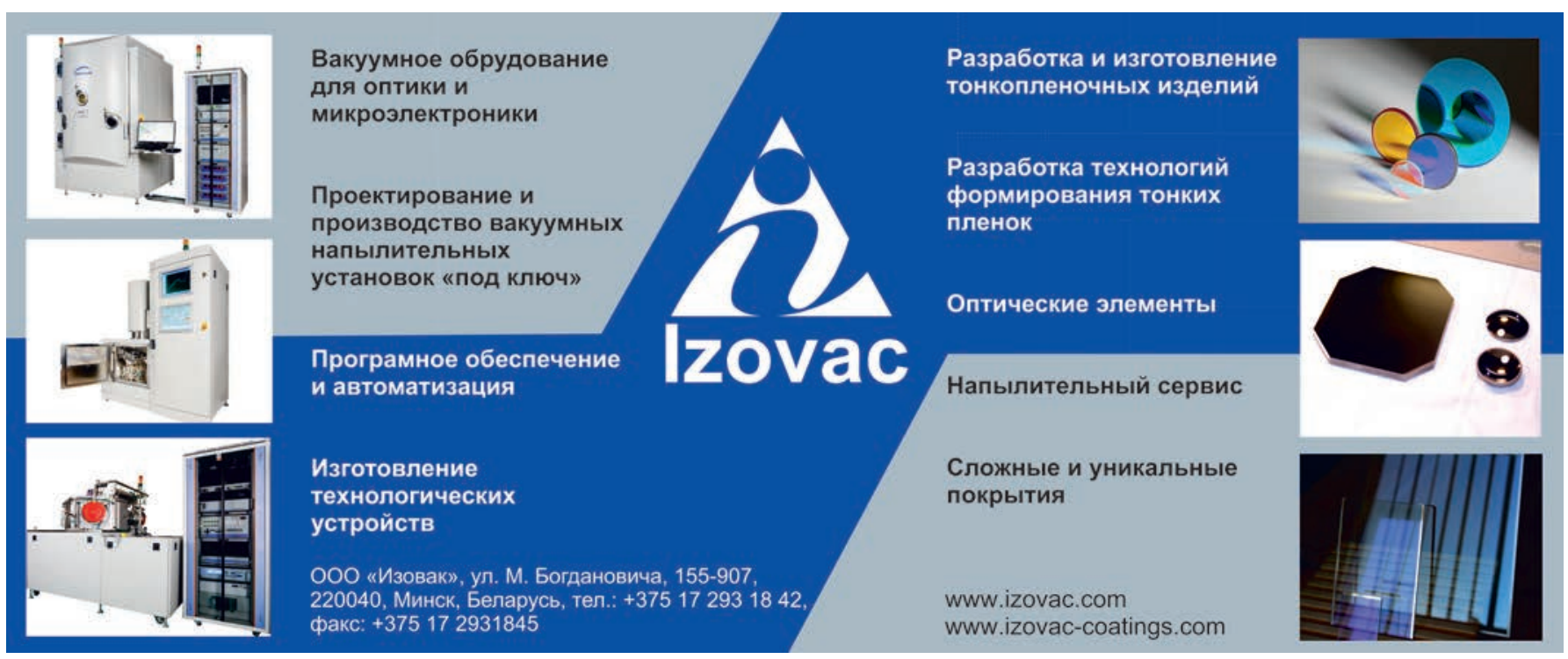

